\title{
Uso del agua de coco (Cocos nucifera) como terapia electrolítica intravenosa en caninos deshidratados
}

\author{
USE OF COCONUT WATER (Cocos nucifera) AS INTRAVENOUS ELECTROLYTIC THERAPY IN \\ DEHYDRATED CANINES
}

\author{
Yamili Mendoza Q. ${ }^{1}$, Olga Li E. ${ }^{2}$, Luis Hoyos S. ${ }^{2}$, Juan Espinoza B. ${ }^{1}$, \\ Pedro Angulo H. ${ }^{1}$, Diego Díaz C. ${ }^{1}$, César Lázaro D. ${ }^{1,3}$
}

\section{Resumen}

\begin{abstract}
El presente trabajo tuvo como objetivo evaluar el uso del agua de coco (AC) aplicado por vía intravenosa en perros. En una primera fase se evaluó la esterilidad e isotonicidad del AC. En una segunda fase se evaluaron los cambios hematológicos producidos 24 horas pos-aplicación del AC por vía intraperitoneal en ratas. En una tercera fase se evaluó la aplicación de $\mathrm{AC}$ vía intravenosa en perros con y sin actividad física $(n=20$, por grupo), donde se determinaron los valores hematológicos, $\mathrm{pH}$ y niveles de cloro y sodio antes y después de su aplicación, así como la frecuencia cardiaca, frecuencia respiratoria y temperatura al inicio, a los 15,30, 45, 60 minutos de iniciada la aplicación y 30 minutos después de la aplicación del AC. Los resultados muestran que el AC es estéril e isotónico, no produjo alteraciones significativas en los valores hematológicos de las ratas ni en los perros sin actividad física. Sin embargo, los perros con actividad física mostraron una reducción del hematocrito a los 30 minutos pos-aplicación y una leve reducción de los niveles de sodio.
\end{abstract}

Palabras clave: agua de coco; perros; valores hematológicos; electrolitos; constantes fisiológicas

\section{AbSTRaCT}

The objective of this study was to evaluate the use of coconut water (AC) applied intravenously in dogs. In a first phase the sterility and isotonicity of the CA was evaluated. In a second phase, the hematological changes produced 24 hours after the application of

\footnotetext{
${ }^{1}$ Laboratorio de Farmacología y Toxicología Veterinaria, Facultad de Medicina Veterinaria, Universidad Nacional Mayor de San Marcos, Lima, Perú

${ }^{2}$ Laboratorio de Patología Clínica y Biología Molecular, Facultad de Medicina Veterinaria, Universidad Nacional Mayor de San Marcos, Lima, Perú

${ }^{3}$ E-mail: clazarod@unmsm.edu.pe
}

Recibido: 29 de agosto de 2017

Aceptado para publicación; 30 de marzo de 2018 
CA by intraperitoneal route in rats were evaluated. In a third phase the application of AC applied intravenously was evaluated in dogs with and without physical activity $(n=20$, per group), where the hematological values, $\mathrm{pH}$ and chlorine and sodium levels were determined before and after its application, as well as the heart rate, respiratory rate and temperature at the beginning, at 15,30, 45, 60 minutes after the start of the application and 30 minutes after the application of the $\mathrm{AC}$. The results showed that $\mathrm{AC}$ is sterile and isotonic, did not produce significant alterations in the hematological values of rats or in dogs without physical activity. However, dogs with physical activity showed a reduction in hematocrit at 30 minutes after application and a slight reduction in sodium levels.

Key words: water coconut; dogs; hematological values; electrolytes; physiological constants

\section{INTRODUCCIÓN}

El coco es el fruto de la palma de cocotero, perteneciente a la familia Palmaceae, género Cocos y especie Cocos nucifera. En el Perú se le puede encontrar en gran parte de la costa norte y selva, siendo cultivada en Piura, Tumbes, la parte tropical de Cerro de Pasco y casi la totalidad de la región San Martín (Monteagudo y Huamán, 2010; Núñez, 2010).

El agua de coco (AC) es un líquido transparente (Fife, 2005), ligeramente hipotónico, más ácido que el plasma y tiene una gravedad específica de aproximadamente 1.020, comparable con el plasma canino de 1.023 (Feldman y Sink, 2008). La composición de electrolitos del AC se asemeja al líquido intracelular, siendo potasio, calcio y magnesio los cationes predominantes, mientras que sodio, cloro y fosfato se encuentran en baja concentración (Kuberski et al., 1979).

El uso del AC como solución de reposición electrolítica por vía intravenosa ha sido reportado en varias especies. Uno de los primeros reportes fue dado en Sri Lanka y en Sumatra, Indonesia, durante la Segunda Guerra Mundial, donde los japoneses y británicos lo usaron en pacientes humanos en situación de emergencia cuando no contaban con soluciones electrolíticas comerciales (Eiseman, 1954; Goldsmith, 1962; Olurin y Durowoju,
1972). En las Islas Salomón se utilizó de forma exitosa en un paciente humano quien recibió $1200 \mathrm{ml} /$ día durante dos días (CampbellFalck et al., 2000). Asimismo, Pérez-García et al. (2010) lograron normalizar la frecuencia respiratoria, el pulso y la temperatura en 10 terneros con signos clínicos de deshidratación con la aplicación intravenosa de AC.

Los perros son susceptibles a deshidratación y desbalances electrolíticos ocasionados por las altas temperaturas ambientales, el inadecuado suministro de agua, el exceso de ejercicio, y por enfermedades gastrointestinales que cursen con vómitos y diarreas, entre otros, pudiendo ser el AC una alternativa frente al uso de sueros electrolíticos convencionales en zonas geográficas donde abunde este fruto. Por ello, el objetivo del estudio fue evaluar el uso del agua de coco como terapia electrolítica intravenosa en perros.

\section{Materiales y Métodos}

\section{Obtención y Extracción del Agua de Coco}

Se adquirieron 80 cocos inmaduros (seis meses desde su inflorescencia hasta la cosecha; EMBRAPA, 2000; IICA, 2001) del Mercado Mayorista N. ${ }^{\circ} 2$ de Frutas en el Distrito de San Luis (Lima, Perú), seleccionando aquellos cuyo exocarpio estuviera libre de rajaduras que pudieran contaminar su 
contenido. Los cocos fueron transportados al Laboratorio de Farmacología y Toxicología Veterinaria de la Facultad de Medicina Veterinaria (FMV) de la Universidad Nacional Mayor de San Marcos (UNMSM) en Lima.

Los frutos fueron lavados en el laboratorio con agua potable, desinfectados con una solución de hipoclorito de sodio (150 ppm), y almacenados a temperatura de refrigeración $\left(4{ }^{\circ} \mathrm{C}\right)$. En los días que se programaba el experimento se realizaba la extracción del AC; para esto, se hacía un corte a nivel del polo germinal con una sierra estéril dejando expuesto el endocarpio el cual fue perforado con ayuda de un punzón estéril (Prades et $a l .$, 2012). Una vez expuesto, el AC fue colectado mediante un equipo de venoclisis (SensiMedical ${ }^{\circledR}$ ) en una bolsa estéril (Changshu Taining Medical Equipment, China) (Figura 1); así mismo, se colectó una muestra con una jeringa estéril de $20 \mathrm{ml}$ para evaluar la esterilidad e isotonicidad. Las bolsas con el AC fueron mantenidas y transportadas a temperatura de refrigeración $\left(4{ }^{\circ} \mathrm{C}\right)$ en un tiempo máximo 2-3 horas hasta el Hospital Veterinario Central del Ejercito (Lima, Perú) donde se encontraban los perros.

\section{Esterilidad e Isotonicidad del Agua de Coco}

La esterilidad del AC fue determinada mediante el conteo de Bacterias Mesófilas Aeróbicas Totales (BMAT) en 10 cocos comerciales. Para esto, se realizaron diluciones seriadas $\left(10^{-1}, 10^{-2}, 10^{-3}\right)$ del AC en agua peptonada y se colocó $1 \mathrm{ml}$ en placas Petri (15x90 mm) junto con $15 \mathrm{ml}$ de Agar Plate Count (Himedia, India). Luego de la homogenización y solidificación en temperatura ambiente, las placas fueron rotuladas e incubadas a $35^{\circ} \mathrm{C}$ durante $48 \mathrm{~h}$, para proceder al recuento de las colonias presentes en las placas. Los resultados fueron expresados en Unidades Formadoras de Colonias por mililitro del producto (Murcia-Mena, 2010).
Para la isotonicidad se adicionaron $9 \mathrm{ml}$ de agua de coco a $1 \mathrm{ml}$ de sangre entera de perro. La mezcla fue colocada en una centrífuga (Gemmy Industrial Corp, Taiwán) a $515 \mathrm{~g}$ por $10 \mathrm{~min}$. Se observaron las características macroscópicas del plasma (hemólisis), teniendo en cuenta que $0.3 \mathrm{~g} / 1$ es la concentración de hemoglobina libre mínima detectable visualmente (Agudelo et al., 2014). Como comparación se realizó una prueba similar con $1 \mathrm{ml}$ de sangre y $9 \mathrm{ml}$ de $\mathrm{NaCl}$ al $0.9 \%$ (Pérez-García et al., 2010). La sangre fue colectada de 10 perros sanos machos mestizos, con edades entre los 3 y 5 años, provenientes de la Clínica de Animales Menores de la FMV-UNMSM.

\section{Evaluación in vivo del Agua de Coco}

Los procedimientos y metodologías fueron revisados y autorizados por el Comité de Ética y Bienestar Animal (CEBA) de la institución mediante Constancia N. ${ }^{\circ}$ 2018-002.

\section{En ratas:}

Se utilizaron 20 ratas albinas macho (Holtzman) de aproximadamente $250 \mathrm{~g}$ de peso vivo y 14 semanas de edad del bioterio de la FMV-UNMSM. Un grupo $(n=10)$ recibió $10 \mathrm{ml}$ de AC y el otro grupo $(\mathrm{n}=10)$ se le aplicó $10 \mathrm{ml}$ de $\mathrm{NaCl}$ 0.9\% (B. Braun Medical, Perú), en ambos casos por vía intraperitoneal. Después de 24 horas, fueron anestesiadas con $30 \mathrm{mg} / \mathrm{kg}$ de pentobarbital sódico (Halatal ${ }^{\circledR}$, Montana, Perú) por vía intraperitoneal y se colectaron muestras de sangre $(2-3 \mathrm{ml})$ mediante punción cardiaca para evaluación de parámetros hematológicos. Las ratas fueron sacrificadas con una dosis de $150 \mathrm{mg} / \mathrm{kg}$ de pentobarbital sódico por vía intraperitoneal (Sainz et al., 1983) y sometidas a necropsia donde se revisó la zona de inoculación en busca de alteraciones macroscópicas que indiquen procesos inflamatorios/infecciosos. 
Las muestras fueron trasladadas en una caja isotérmica con temperatura de $4{ }^{\circ} \mathrm{C}$ al Laboratorio de Patología Clínica Veterinaria de la FMV-UNMSM. La concentración de hemoglobina fue determinada por espectrofotometría mediante el método de cianometahemoglobina, el hematocrito por el método de microhematocrito, el recuento de eritrocitos y leucocitos mediante el hemocitómetro de Neubauer y el recuento de trombocitos se realizó por extendido sanguíneo y tinción Wright. Asimismo, se obtuvieron los siguientes índices hematimétricos: volumen corpuscular medio [(hematocrito/ recuento de eritrocitos) $x$ 100], hemoglobina corpuscular media [(hemoglobina $\mathrm{x}$ 10)/ recuento de eritrocitos] y concentración de hemoglobina corpuscular media [(hemoglobina/ hematocrito) $\mathrm{x}$ 100].

\section{En perros:}

Se utilizaron 40 perros adultos del Hospital Veterinario Central del Ejercito (Lima, Perú) con edades entre 2 y 6 años, hembras y machos, clínicamente sanos, sin antecedentes de presentación de enfermedades en el último año, y que recibían una dieta balanceada (Cambo ${ }^{\circledR}$, Rintisa, Perú) y agua ad libitum.

En una primera parte se utilizaron 20 perros que no realizaron ningún tipo de actividad física previa y a los cuales se les aplicó el AC por vía intravenosa, el cual fue extraído de 60 cocos. Para el cálculo del volumen de aplicación se utilizó la siguiente fórmula: Volumen de agua de coco $(\mathrm{ml})=$ deshidratación (\%) x peso (kg) x 10 (Rojas et al., 2009). El porcentaje de deshidratación fue estimado con base a parámetros clínicos como pérdida de elasticidad cutánea, mucosas secas, globos oculares hundidos, y aumento del tiempo de llenado capilar (Blood y Radostits, 1998). En esta etapa y debido a que los perros no realizaron actividad física se consideró que tenían 1\% de deshidratación. El AC fue co- locado a través de la vena cefálica por intermedio de un catéter flexible (Cath Pro ${ }^{\circledR}$, MediFirst, Francia) durante 90 minutos.

Antes de la aplicación se tomó una muestra de sangre en un tubo con K2-EDTA (BD Vacutainer ${ }^{\circledR}$, EEUU) como muestra control. Una vez aplicado el AC, se retiró el catéter y los animales permanecieron en observación por $24 \mathrm{~h}$ en caniles. Luego del periodo de observación, se tomó una muestra de sangre adicional. Las determinaciones hemáticas se realizaron siguiendo los lineamientos indicados para la evaluación hematológica en ratas.

En una segunda etapa se utilizaron 20 perros sometidos a actividad física, la cual consistió en una serie de ejercicios como caminar, correr y realizar saltos de ataque por 30 minutos, los cuales formaban parte del programa de entrenamiento del «Curso de Guías y Adiestramiento de Canes de Seguridad» impartido por el personal del Ejército Peruano. Concluido el entrenamiento, se determinó el grado de deshidratación $(<5 \%)$ para el cálculo de volumen de AC a aplicar conforme lo descrito en la primera etapa (Figura 1). Luego de la aplicación, los animales permanecieron en observación en los caniles. En este caso se tomaron muestras de sangre a los 30 min y a las $24 \mathrm{~h}$ de la aplicación del AC.

En esta etapa se midieron, además, las siguientes constantes fisiológicas: temperatura rectal, frecuencia cardiaca y frecuencia respiratoria antes de la aplicación del AC (T0), a los 15 (T1), 30 (T2), 45 (T3) y 60 (T4) min durante la aplicación del AC, y a los $30 \mathrm{~min}$ (T5) posteriores a la aplicación del AC. Las muestras de sangre fueron procesadas en forma similar a las muestras de los perros sin actividad física, pero se evaluó el hematocrito, así como los niveles de $\mathrm{pH}$, sodio $(\mathrm{Na}+)$ y cloro (Cl-) mediante un analizador de electrolitos automatizado (ABL80 Flex, Radiometer, Dinamarca). 


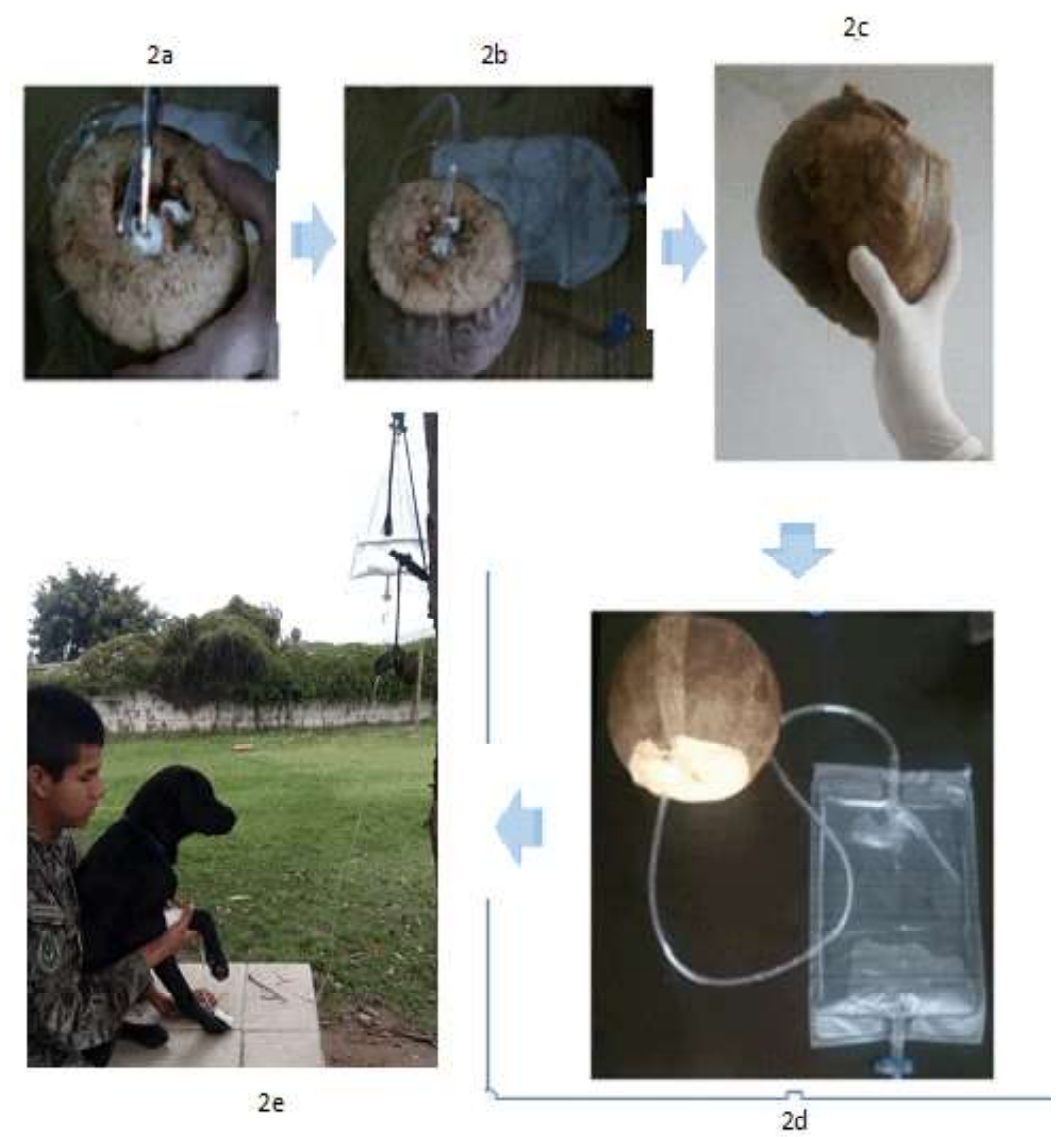

Figura 1. Extraccion y apılicacion intravenosa del agua de coco en perros del Hospital Central Veterinario del Ejército. (2a) Exposición del endocarpio. (2b) Punción del poro germinal funcional. (2c, 2d) Recolección del agua de coco en una bolsa estéril. 2e. Aplicación intravenosa del agua de coco en un perro

\section{Resultados y Discusión}

La evaluación in vitro indicó que no hubo crecimiento bacteriano lo cual demuestra la esterilidad del AC y las medidas correctas de higiene durante el proceso de extracción del agua (Pérez-García et al., 2010). Asimismo, no se detectó hemólisis al mezclar la sangre con el AC, lo cual indica una tonicidad similar al plasma y al cloruro de sodio al $0.9 \%$. Este resultado es similar al presentado en un estudio en terneros, donde se evaluó sangre antes y después $(60 \mathrm{~min})$ de la aplicación de $50 \mathrm{ml}$ de agua de coco por vía intravenosa y no se observó hemólisis (Pérez-García et al., 2010).
Las ratas tratadas con AC no mostraron diferencias significativas para los valores hematológicos, exceptuando el recuento de trombocitos los cuales se encontraban por encima del valor de referencia (Cuadro 1). Las ratas, por otro lado, no presentaron signos clínicos ni alteraciones a la necropsia que sean compatibles con una trombocitosis patológica. Tampoco se encontraron alteraciones macroscópicas en la zona de inoculación que indiquen procesos inflamatorios o infecciosos.

En la evaluación en perros sin actividad física no se observaron diferencias significativas en los parámetros en estudio al comparar los valores antes y después de la adminis- 
Cuadro 1. Valores hematológicos (promedio \pm desviación estándar) en ratas albinas (Holtzman) a las 24 horas de la aplicación intraperitoneal de $\mathrm{NaCl} 0.85 \%$ (control) y agua de coco (tratamiento)

\begin{tabular}{llccc}
\hline Parámetro & Unidad & $\begin{array}{c}\text { Control } \\
(\mathrm{n}=10)\end{array}$ & $\begin{array}{c}\text { Tratamiento } \\
(\mathrm{n}=10)\end{array}$ & $\begin{array}{c}\text { Valores de } \\
\text { referencia }^{1}\end{array}$ \\
\hline Eritrocitos & $10^{6} / \mu 1$ & $6.93 \pm 0.86^{\mathrm{a}}$ & $6.74 \pm 0.65^{\mathrm{a}}$ & $6.33-8.64$ \\
Hemoglobina & $\mathrm{g} / \mathrm{dl}$ & $15 \pm 0.62^{\mathrm{a}}$ & $14 \pm 0.64^{\mathrm{a}}$ & $12.09-15.18$ \\
Hematocrito & $\%$ & $44 \pm 1.83^{\mathrm{a}}$ & $43 \pm 1.58^{\mathrm{a}}$ & $33.1-45.8$ \\
$\mathrm{VCM}$ & $\mathrm{fl}$ & $58.77 \pm 6.54^{\mathrm{a}}$ & $58.82 \pm 3.87^{\mathrm{a}}$ & $52.4-59,9$ \\
$\mathrm{HCM}$ & $\mathrm{pg}$ & $21.41 \pm 2.18^{\mathrm{a}}$ & $21.32 \pm 1.26^{\mathrm{a}}$ & $15.6-21,2$ \\
$\mathrm{CHCM}$ & $\mathrm{g} / \mathrm{dl}$ & $33.27 \pm 0.07^{\mathrm{a}}$ & $33.26 \pm 0.08^{\mathrm{a}}$ & $28.1-93.3$ \\
Leucocitos & $10^{3} / \mu 1$ & $8.32 \pm 3.30^{\mathrm{a}}$ & $9.03 \pm 1.82^{\mathrm{a}}$ & $4.84-12.96$ \\
Trombocitos & $10^{5} / \mu 1$ & $5.24 \pm 3.09^{\mathrm{b}}$ & $9.93 \pm 4.69^{\mathrm{a}}$ & $4.28-8.57$ \\
\hline
\end{tabular}

VCM: volumen corpuscular medio; HCM: hemoglobina corpuscular media; CHCM: concentración de hemoglobina corpuscular media

$a, b$ Letras diferentes dentro de filas indican diferencia estadística significativa $(p<0.05)$

${ }^{1}$ Fuente: León et al. (2011) para ratas macho de 9 a 14 semanas de edad

Cuadro 2. Valores hematológicos (promedio \pm desviación estándar) antes y 24 horas después de la aplicación endovenosa de agua de coco vía endovenosa en 20 perros adultos que no realizaron actividad física previa

\begin{tabular}{llccc}
\hline Parámetro & Unidad & Antes & $\begin{array}{c}\text { Después } \\
(24 \text { horas })\end{array}$ & $\begin{array}{c}\text { Valores de } \\
\text { referencia }^{1}\end{array}$ \\
\hline Eritrocitos & $10^{6} / \mu 1$ & $5.84 \pm 1.10^{\mathrm{a}}$ & $5.98 \pm 1.10^{\mathrm{a}}$ & $5.7-9$ \\
Hemoglobina & $\mathrm{g} / \mathrm{dl}$ & $14.65 \pm 1.49^{\mathrm{a}}$ & $14.85 \pm 1.71^{\mathrm{a}}$ & $13.8-20.4$ \\
Hematocrito & $\%$ & $44 \pm 4.46^{\mathrm{a}}$ & $44.55 \pm 5.13^{\mathrm{a}}$ & $42-62$ \\
$\mathrm{VCM}$ & $\mathrm{fl}$ & $76.98 \pm 10.34^{\mathrm{a}}$ & $76.68 \pm 14.38^{\mathrm{a}}$ & $62.7-74.6$ \\
$\mathrm{HCM}$ & $\mathrm{pg}$ & $25.62 \pm 3.41^{\mathrm{a}}$ & $25.55 \pm 1.85^{\mathrm{a}}$ & $20.5-24.8$ \\
$\mathrm{CHCM}$ & $\mathrm{g} / \mathrm{dl}$ & $33.28 \pm 0.18^{\mathrm{a}}$ & $33.32 \pm 0.06^{\mathrm{a}}$ & $31.6-34.4$ \\
Leucocitos & $10^{3} / \mu \mathrm{l}$ & $13.95 \pm 5.90^{\mathrm{a}}$ & $13.79 \pm 4.10^{\mathrm{a}}$ & $5.8-20.3$ \\
Trombocitos & $10^{5} / \mu 1$ & $2.50 \pm 0.15^{\mathrm{a}}$ & $2.67 \pm 0.15^{\mathrm{a}}$ & $1.73-4.87$ \\
\hline
\end{tabular}

VCM: volumen corpuscular medio; HCM: hemoglobina corpuscular media; CHCM: concentración de hemoglobina corpuscular media

$a, b$ Letras diferentes dentro de filas indican diferencia estadística significativa $(p<0.05)$

${ }^{1}$ Fuente: Moritz et al. (2004)

tración intravenosa del AC (Cuadro 2). Esto podría estar relacionado a las propiedades del $\mathrm{AC}$, ya que es una solución muy parecida al plasma sanguíneo (Feldman y Sink, 2008).
En la evaluación en perros con actividad física se presentaron niveles elevados de $\mathrm{pH}$ en sangre (Cuadro 3), posiblemente por la mayor demanda de oxígeno que produce 
Cuadro 3. Evaluación plasmática antes, 30 minutos y 24 horas después de finalizada la aplicación intravenosa de agua de coco en 20 perros adultos que realizaron actividad física (promedio \pm desviación estándar)

\begin{tabular}{lcccc}
\hline Parámetro & Antes & $\begin{array}{c}30 \mathrm{~min} \\
\text { después }\end{array}$ & $24 \mathrm{~h}$ después & $\begin{array}{c}\text { Valores de } \\
\text { referencia* }\end{array}$ \\
\hline $\mathrm{pH}$ & $7.63 \pm 0.14^{\mathrm{a}}$ & $7.61 \pm 0.16^{\mathrm{a}}$ & $7.59 \pm 0.19^{\mathrm{a}}$ & $7.31-7.53$ \\
Hematocrito (\%) & $44.65 \pm 5.63^{\mathrm{a}}$ & $40.6 \pm 7.01^{\mathrm{b}}$ & $44.55 \pm 5.13^{\mathrm{a}}$ & $41-58$ \\
Sodio (mmol/l) & $135.7 \pm 7.15^{\mathrm{b}}$ & $130.85 \pm 8.37^{\mathrm{a}}$ & $128.65 \pm 6.12^{\mathrm{a}}$ & $137-149$ \\
Cloro (mmol/l) & $126.08 \pm 4.94^{\mathrm{a}}$ & $124.75 \pm 6.02^{\mathrm{a}}$ & $126.2 \pm 7.29^{\mathrm{a}}$ & $99-110$ \\
\hline
\end{tabular}

${ }_{a, b}$ Letras diferentes dentro de filas indican diferencia estadística significativa $(\mathrm{p}<0.05)$

${ }^{1}$ Fuente: Campbell y Chapman (2000)

Cuadro 4. Evaluación de las constantes fisiológicas (promedio \pm desviación estándar) antes, durante y después de la aplicación intravenosa de agua de coco (AC) en 20 perros adultos que realizaron actividad física

\begin{tabular}{|c|c|c|c|c|c|c|c|}
\hline \multirow{2}{*}{$\begin{array}{l}\text { Constante } \\
\text { fisiológica }\end{array}$} & \multicolumn{6}{|c|}{$\begin{array}{l}\text { Momento de la evaluación }{ }^{1} \\
\text { (minutos) }\end{array}$} & \multirow{2}{*}{$\begin{array}{l}\text { Valores de } \\
\text { referencia }^{2}\end{array}$} \\
\hline & T0 & $\mathrm{T} 1$ & $\mathrm{~T} 2$ & T3 & $\mathrm{T} 4$ & T5 & \\
\hline $\begin{array}{l}\text { Frecuencia } \\
\text { cardiaca } \\
\text { (lat } / \mathrm{min})\end{array}$ & $\begin{array}{c}93.75 \pm \\
21.96\end{array}$ & $\begin{array}{l}89.8 \pm \\
22.49\end{array}$ & $\begin{array}{l}84.5 \pm \\
24.79\end{array}$ & $\begin{array}{l}86.6 \pm \\
26.27\end{array}$ & $\begin{array}{c}83.85 \pm \\
25.01\end{array}$ & $\begin{array}{c}85.45 \pm \\
22.16\end{array}$ & $70-120$ \\
\hline $\begin{array}{l}\text { Frecuencia } \\
\text { respiratoria } \\
\text { (resp/min) }\end{array}$ & $\begin{array}{c}95.95 \pm \\
54.06\end{array}$ & $\begin{array}{c}95.05 \pm \\
46.60\end{array}$ & $\begin{array}{c}81.8 \pm \\
52.11\end{array}$ & $\begin{array}{c}75.95 \pm \\
48.29\end{array}$ & $\begin{array}{l}75.3 \pm \\
47.31\end{array}$ & $\begin{array}{c}82.85 \pm \\
48.23\end{array}$ & $18-34$ \\
\hline $\begin{array}{l}\text { Temperatura } \\
\text { rectal }\left({ }^{\circ} \mathrm{C}\right)\end{array}$ & $\begin{array}{c}39.24 \pm \\
0.52\end{array}$ & $\begin{array}{c}39.16 \pm \\
0.69\end{array}$ & $\begin{array}{c}39.0 \pm \\
0.79\end{array}$ & $\begin{array}{c}38.9 \pm \\
0.75\end{array}$ & $\begin{array}{c}38.82 \pm \\
0.63\end{array}$ & $\begin{array}{c}38.88 \pm \\
0.63\end{array}$ & $\begin{array}{c}38.9 \\
\text { (media) }\end{array}$ \\
\hline
\end{tabular}

${ }^{1}$ TO: antes de la aplicación de AC; T1-T4: 15, 30, 45, 60 minutos durante la aplicación de AC; T5: 30 minutos posteriores a la aplicación de AC

${ }^{2}$ Fuente: Merck y Merial (2007)

una respuesta de hiperventilación, la cual disminuye la $\mathrm{pCO}_{2}$ y aumenta el $\mathrm{pH}$ (Forero et al., 2006). Por otro lado, se observó una disminución significativa del hematocrito $(\mathrm{p}<0.05)$ a los $30 \mathrm{~min}$ de haber finalizado la aplicación intravenosa de AC, debido principalmente al incremento del volumen plasmático, el cual fue restablecido a las 24 horas gracias a los mecanismos fisiológicos de regulación hídrica. También hay que considerar que, a pesar de la aplicación parenteral, todos los perros fueron a beber agua al finalizar la aplicación del AC, pues mantenían la sensación de sed luego del ejercicio realizado, a pesar de haberse restablecido la osmolalidad plasmática (Guyton y Hall, 2011). Además, la temperatura ambiental $\left(>28^{\circ} \mathrm{C}\right)$ influyó en la necesidad del consumo de agua. 
En relación a los electrolitos, se observó una significativa, aunque ligera reducción de los niveles de sodio (hiponatremia) posaplicación $(\mathrm{p}<0.05)$, lo cual podría atribuirse a su baja concentración en el AC. Por otro lado, no se apreciaron diferencias significativas en los valores de cloro, a pesar que durante todo el experimento se apreció un estado de hipercloremia. Pérez-García et al. (2010) encontraron que el AC contribuyó a la homeostasis, llevando a la normalidad los valores de sodio y potasio y favoreciendo los iones de calcio hemático. Según Latimer et al. (2005), los perros de carrera pueden sufrir hiponatremia e hipocalemia a pesar de no sudar. Sin embargo, se desconoce cuál es el mecanismo que explica la pérdida de estos electrolitos de forma excesiva. Estos investigadores indican como posible explicación el ejercicio continuo en perros con episodios de hiponatremia, debido que la hiperventilación es usada como medio de enfriamiento y mediante este mecanismo se pierde $\mathrm{Na}+\mathrm{y}$ $\mathrm{NaCO}_{3}$ a través de la saliva. Por otro lado, la hipercloremia podría explicarse por la pérdida de $\mathrm{NaHCO}_{3}$ a través de la orina y la hiperventilación, eventos que causan un incremento relativo del cloro sérico o plasmático, aunque haya un déficit de cloro corporal total en caso de deshidratación.

En el Cuadro 4 se presentan los resultados de las constantes fisiológicas en perros sometidos a actividad física y suplementados con AC. La frecuencia cardiaca se mantuvo dentro del rango normal durante todo el proceso, la frecuencia respiratoria estuvo muy acelerada y la temperatura corporal se mantuvo cerca al límite superior, posiblemente debido a la temperatura ambiental del verano $\left(>28^{\circ} \mathrm{C}\right)$. Es importante señalar que la sujeción del animal para la colocación del catéter intravenoso provoca estrés en el animal, lo que puede explicar los valores de frecuencia cardiaca, que a pesar de estar dentro del rango normal, fueron ligeramente altos en un inicio para luego reducirse progresivamente conforme el animal se tranquilizaba. La temperatura corporal está ligada a la frecuencia respiratoria, pues los perros utilizan el jadeo para reducir su temperatura mediante la evaporación de agua de la lengua y la inhalación de aire (Forero et al., 2006). Además, durante el ejercicio se libera la energía contráctil del músculo, la cual finalmente se convierte en calor corporal, y se aumenta el consumo de oxígeno, liberando calor en los tejidos corporales internos (Guyton y Hall, 2011).

\section{Conclusiones}

El agua de coco es un líquido estéril e isotónico que no provocó cambios significativos a nivel sanguíneo a las 24 horas de la aplicación, no influyó en el pH ni en los niveles de cloro, aunque redujo ligera, pero significativamente los niveles de sodio.

\section{Literatura Citada}

1. Agudelo M, Ortega V, Olivera-Ángel M. 2014. Efecto de la hemólisis de la muestra de sangre canina en el resultado de algunos analitos enzimáticos. Rev Biosalud 13(1): 21-29.

2. Blood DC, Radostits OM. 1998. Medicina veterinaria. $7^{\circ}$ ed. México: McGraw Hill. 1502 p.

3. Campbell A, Chapman M. 2000. Handbook of poisoning in dogs and cats. Oxford: Blackwell Science. 284 p.

4. Campbell-Falck, Thomas T, Falck TM, Tutuo N, Clem K. 2000. The intravenous use of coconut water. Am J Emerg Med 18: 108-111. doi: 10.1016/ S0735-6757(00)90062-7

5. [EMBRAPA] Empresa Brasileira de Pesquisa Agropecuária. 2000. Técnicas para colheita e pós-colheita de coco verde. Brasil: EMBRAPA Semi-Árido. Comunicado Técnico $95.6 \mathrm{p}$.

6. Eiseman B. 1954. Intravenous infusion of coconut water. AMA Arch Surg 68: 167-178. doi: 10.1001/archsurg.1954.01260050169005 
7. Feldman BF, Sink CA. 2008. Practical transfusion medicine for small animal practitioner. USA: Teton New Media. 140 p.

8. Fife B. 2005. Coconut cures. Preventing and treating common health problems with coconut. USA: Colorado Spring. $256 \mathrm{p}$.

9. Forero JH, Lozano PA, Camargo BO. 2006. Parámetros fisiológicos en caninos pre y post competencia de Agility en Bogotá, Colombia. Rev Med Vet 12: 57-71.

10. Goldsmith HS. 1962. Coconut water for intravenous therapy. Brit J Surg 49: 421-422. doi: 10.1002/bjs. 18004921617

11. Guyton A, Hall J. 2011. Tratado de fisiología médica. $12^{\circ}$ ed. España: Elsevier. $1092 \mathrm{p}$.

12. [IICA] Instituto Interamericano de Cooperación para la Agricultura. 2001. Guía técnica del cultivo de coco. El Salvador: IICA. 53 p.

13. Kuberski T, Roberts A, Linehan B, Bryden RN, Teburae M. 1979. Coconut water as a rehydration fluid. $\mathrm{N}$ Z Med J 90: 98-100.

14. Latimer K, Mahaffey $E$ y Prasse K. 2005. Duncan \& Prasse's veterinary laboratory medicine clinical pathology. $5^{\text {th }}$ ed. USA: Wiley-Blackwell. 609 p.

15. León A, Blanco D, Peña A, Ronda M, González B, Arteaga M, Bada A, González, Y, Mancebo A. 2011. Valores hematológicos y bioquímicos de las ratas Sprague Dawley Producidas en CENPALAB, Cenp: SPRD. REDVET 12(11). [Internet]. Disponible en: http:// www.veterinaria.org/revistas/redvet/ n111111/111101.pdf

16. Merck Publishing and Merial Staff. 2007. The Merck/Merial manual for pet health. USA: Elsevier. $1300 \mathrm{p}$.

17. Moritz A, Fickenscher Y, Meyer K, Failing K, Weiss D. 2004. Canine and feline hematology reference values for ADVIA 120 hematology system. Vet Clin Pathol 33: 32-38. doi: 10.1111/j.1939165X.2004.tb00347.x
18. Monteagudo A, Huamán M. 2010. Catálogo de los árboles y afines de la Selva Central del Perú. Arnaldoa 17: 203-242.

19. Murcia-Mena ER. 2010. Evaluación de la calidad microbiológica del agua de coco envasada en presentación de un litro, registrada y comercializada en el distrito ${ }^{\circ} .^{\circ} 2$ del área metropolitana de San Salvador. Tesis de Químico Farmacéutico. San Salvador: Univ. de El Salvador. $153 \mathrm{p}$.

20. Núñez, R. 2010. Estudio tecnológico del componente fibra de dos variedades de coco enano (Cocos nucifera) de los distritos de Lamas, Tarapoto y Pucacaca en la Región San Martín. Tesis de Ingeniero Agroindustrial. Tarapoto: Univ. Nacional de San Martín. 127 p.

21. Olurin EO, Durowoju JE. 1972. Intravenous coconut water therapy in surgical practice. West Afr Med J Niger Med Dent Pract 21: 124-131.

22. Pérez-García C, Benitez R, Cuesta M, Silveira E. 2010. Utilización del agua de coco por vía intravenosa como terapia alternativa en la deshidratación en terneros. REDVET 11(03B). [Internet]. Disponible en: http://www.veterinaria.org/revistas/redvet/n030310B/ 0310B_TL05.pdf

23. Prades A, Dornier M, Diop N, Pain J. 2012. Coconut water preservation and processing: a review. Fruits 67: 157-171. doi: 10.1051/fruits/2012009

24. Rojas I, Castro M, Chacón L, De Pablos J, López N, Urbina-Medina $H$. 2009. Hidratación parenteral. Arch Venez Puer Pediatr 72: 154-162.

25. Sainz L, García de Osma JL, Compaire C. 1983. Métodos eutanásicos especialmente recomendados en los animales de laboratorio. En: Animales de laboratorio: (producción, manejo y control sanitario). Madrid: Instituto Nacional de Investigaciones Agrarias. p 253-258. 